\title{
A selection of recent original research papers
}

\author{
Prem Soman, MD, PhD
}

\author{
Improved Cardiac Risk Assessment with Noninvasive \\ Measures of Coronary Flow Reserve \\ Boston, MA \\ Murthy V, Naya M, Foster CR, Hainer J, Gaber M, Di \\ Carli G, Blankstein R, Dorbala S, Sitek A, Pencina MJ, \\ Di Carli MF \\ Circulation 2011; 124: 2215
}

Context Impaired vasodilator function is an early manifestation of coronary artery disease and may precede angiographic stenosis. It is unknown whether noninvasive assessment of coronary vasodilator function in patients with suspected or known coronary artery disease carries incremental prognostic significance.

Methods and Results A total of 2,783 consecutive patients referred for rest/stress positron emission tomography were followed up for a median of 1.4 years (interquartile range 0.7-3.2 years). The extent and severity of perfusion abnormalities were quantified by visual evaluation of myocardial perfusion images. Rest and stress myocardial blood flows were calculated with factor analysis and a 2-compartment kinetic model and were used to compute coronary flow reserve (coronary flow reserve equals stress divided by rest myocardial blood flow). The primary end point was cardiac death. Overall 3-year cardiac mortality was $8.0 \%$. The lowest tertile of coronary flow reserve $(<1.5)$ was associated with a 5.6-fold increase in the risk of cardiac death $(95 \%$ confidence interval, 2.5-12.4; $P<.0001$ ) compared with the highest tertile. Incorporation of coronary flow reserve into cardiac death risk assessment models resulted in an increase in the c index from 0.82 (95\% confidence interval $0.78-0.86$ ) to 0.84 (95\% confidence interval $0.80-0.87 ; \quad P=.02)$ and in a net reclassification improvement of 0.098 (95\% confidence interval 0.025 0.180 ). Addition of coronary flow reserve resulted in

From the Cardiovascular Institute, University of Pittsburgh, Pittsburgh, PA.

Reprint requests: Prem Soman, $\mathrm{MD}, \mathrm{PhD}$, Cardiovascular Institute, University of Pittsburgh, Pittsburgh, PA; somanp@upmc.edu. J Nucl Cardiol 2012;19:1-4.

$1071-3581 / \$ 34.00$

Copyright $(\subset 2011$ American Society of Nuclear Cardiology.

doi:10.1007/s12350-011-9501-x correct reclassification of $34.8 \%$ of intermediate-risk patients (net reclassification improvement $=0.487$; 95\% confidence interval 0.262-0.731). Corresponding improvements in risk assessment for mortality from any cause were also demonstrated. Thus, noninvasive quantitative assessment of coronary vasodilator function with positron emission tomography is a powerful, independent predictor of cardiac mortality in patients with known or suspected coronary artery disease and provides meaningful incremental risk stratification over clinical and gated myocardial perfusion imaging variables.

Significance The incremental prognostic value of CFR over other clinical, functional, and perfusion variables probably reflects its involvement very early on in atherosclerosis. The additive prognostic value of CFR assessment was also present in patient with visually normal myocardial perfusion.

Silent Myocardial Ischemia and Long-Term Coronary Artery Disease Outcomes in Apparently Healthy People from Families with Early Onset Ischemic Heart Disease

Baltimore, $M D$

Kral BG, Becker LC, Vaidya D, Yanke LR, Becker DM European Heart Journal 2011; 32: 2766-2772

Context The prevalence of myocardial ischemia and its association with long-term incident CAD in apparently healthy siblings of early onset CAD patients is unknown.

Methods and Results Asymptomatic siblings ( $\mathrm{n}=1,287$, aged 30-59 years) of patients with onset of $\mathrm{CAD}<60$ years of age underwent risk factor screening and maximal graded treadmill testing with nuclear perfusion imaging, and were followed for incident $\mathrm{CAD}$ events for up to 25 years. Incident CAD occurred in $15.2 \%$ of siblings ( $68 \%$ acute coronary syndromes); mean time to first $\mathrm{CAD}$ event was $8.2 \pm 5.2$ years. Inducible ischemia was highly prevalent in male siblings (26.9\%), and was independently associated with incident CAD. Male siblings $\geq 40$ years of age who were low or intermediate risk by traditional risk assessment, had a prevalence of inducible ischemia and a 10-year risk of incident $\mathrm{CAD}$ that were near or $\geq 20 \%$. In female siblings $\geq 40$ years of age, the presence of inducible ischemia was also independently associated with incident CAD, but the prevalence of inducible ischemia was markedly lower, as was the risk of incident CAD. Thus, inducible ischemia is highly prevalent in male siblings, suggesting a previously 
unknown long quiescent period before the occurrence of a clinical event. While inducible ischemia is associated with a worse prognosis, male siblings with negative tests still bear a high risk of incident disease, such that we propose that in male siblings over 40 years of age, aggressive primary prevention interventions be instituted without nuclear testing. For women, the prevalence of ischemia was so low as to not warrant screening, but the incidence of CAD was high enough to at least warrant lifestyle interventions.

Significance Although this study indicates that silent ischemia is prevalent in male siblings of prematureCAD patients, and associated with incident CAD, the results do not justify screening myocardial perfusion imaging in this population.

Clinical Value of Absolute Quantification of Myocardial Perfusion with O-15 Water in Coronary Artery Disease

Turku, Finland

Kajander SA, Joutsiniemi EJ, Saraste M, Pietila M, Ukkonen H, Saraste A, Sipila HT, Teras M, Maki M, Airaksinen J, Hartiala J, Knuuti J

Circ Cardiovasc Imaging 2011; 4: 678-648

Context The authors compared relative and absolute myocardial perfusion assessment using O-15 water PET in an intermediate likelihood population for the detection of significant CAD defined by invasive coronary arteriography and FFR measurements.

Methods and Results One hundred four patients with moderate $(30 \%-70 \%)$ pretest likelihood of coronary artery disease (CAD) underwent PET imaging during adenosine stress using ${ }^{15} \mathrm{O}$-water and dynamic imaging. Absolute myocardial blood flow was calculated from which both standard relative myocardial perfusion images and images scaled to a known absolute scale were produced. The patients and the regions then were classified as normal or abnormal and compared against the reference of conventional angiography with fractional flow reserve. In patient-based analysis, the positive predictive value, negative predictive value, and accuracy of absolute perfusion in the detection of any obstructive CAD were $86 \%, 97 \%$, and $92 \%$, respectively, with absolute quantification. The values with relative analysis were $61 \%, 83 \%$, and $73 \%$, respectively. In region-based analysis, the receiver operating characteristic curves confirmed that the absolute quantification was superior to relative assessment. In particular, the specificity and positive predictive value were low using just relative differences in flow. Only 9 of 24 patients with 3-vessel disease were correctly assessed using relative analysis.
Significance This study again shows the superiority of absolute myocardial blood flow measurement over relative perfusion assessment. Although O-15 water could be considered the "gold standard" perfusion tracer, there are several logistic and technical issues which limit its widespread use, and gating cannot be performed. Nevertheless, the major findings of this study can probably be applied to other PET tracers which facilitate absolute flow quantitation. Interestingly, despite being a diffusible tracer with a "near-perfect", flow-uptake relationship, nonlinear, heterogeneous myocardial uptake at high flows still caused false-positive perfusion abnormalities on relative perfusion assessment.

\section{Myocardial Uuptake of $7^{\prime}$-(Z)- ${ }^{123}$ Iodorotenone During Vasodilator Stress in Dogs with Critical Coronary Stenosis}

Charlottesville, VA; Berkeley, CA; Sacramento, CA; San Francisco, $C A$

Broisat A, Ruiz M, Goodman NC, Hanrahan SM, Reutter BW, Brennan KM, Janabi M, Schaefer S, Watson DD, Beller GA, VanBrocklin HF, Glover DK Circ Cardiovasc Imaging 2011; 4: 685-692

Context Radiotracers that target complex I of the mitochondrial electron transport chain have been proposed as a new class of myocardial perfusion imaging agents. 7-(Z)- $\left[{ }^{123}\right.$ I] iodorotenone $\left({ }^{123}\right.$ I-ZIROT), a singlephoton emission computed tomography (SPECT) tracer has demonstrated superior myocardial extraction and retention characteristics in rats and in isolated perfused rabbit hearts. The objective of this study was to fully characterize the biodistribution and myocardial extraction versus flow relationship of ${ }^{123}$ I-ZIROT in an intact large-animal model.

Methods and Results The ${ }^{123}$ I-ZIROT was administered during adenosine $\mathrm{A}_{2 \mathrm{~A}}$ agonist-induced hyperemia in 5 anesthetized dogs with critical left anterior descending (LAD) stenoses. When left circumflex (LCx) flow was maximal, ${ }^{123}$ I-ZIROT and microspheres were coinjected and the dogs were euthanized 5 minutes later. ${ }^{123}$ I-ZIROT biodistribution was evaluated in 2 additional dogs by in vivo planar imaging. At ${ }^{123}$ I-ZIROT injection, transmural LAD flow was unchanged from baseline (mean \pm SEM, $0.90 \pm 0.22$ vs $0.87 \pm 0.11 \mathrm{~mL} /[$ minute $\cdot \mathrm{g}] ; P=.92)$, whereas LCX zone flow increased significantly $($ mean $\pm \mathrm{SEM}, 3.25 \pm 0.51$ vs $1.00 \pm 0.17 \mathrm{~mL} /[\mathrm{min} \cdot \mathrm{g}]$; $P<.05)$. Myocardial ${ }^{123}$ I-ZIROT extraction tracked regional myocardial flow better than either thallium-201 or ${ }^{99 \mathrm{~m}} \mathrm{Tc}$-sestamibi from previous studies using a similar model. Furthermore, the ${ }^{123}$ I-ZIROT LAD/LCx activity ratios by ex vivo imaging or well counting (mean \pm SEM, 
$0.42 \pm 0.08$ and $0.45 \pm 0.1$, respectively) only slightly underestimated the $\mathrm{LAD} / \mathrm{LCx}$ microsphere flow ratio $(0.32 \pm 0.09)$. Thus, the ability of ${ }^{123}$ I-ZIROT to more linearly track blood flow over a wide range makes it a promising new SPECT myocardial perfusion imaging agent with potential for improved coronary artery disease detection and better quantitative estimation of the severity of flow impairment.

Significance This paper describes the flow-uptake relationship of a new, I-123-based myocardial perfusion tracer. First-pass extraction fraction was not determined.

\section{Impact of Cardiac Hybrid Single-Photon Emission Computed Tomography/Computed Tomography Imaging on Choice of Treatment in Coronary Artery Disease}

Zurich, Switzerland

Pazhennkottil AP, Nkoulou, RN, Ghadri J, Herzog BA, Küest SM, Husmann L, Wolfrum M, Goetti R, Buechel RR, Gaemperli O, Lüscher TF, Kaufmann PA

European Heart Journal 2011; 32: 2824-2829

Context Cardiac hybrid imaging by fusing singlephoton emission computed tomography (SPECT) myocardial perfusion imaging with coronary computed tomography angiography (CCTA) provides important complementary diagnostic information for coronary artery disease (CAD) assessment. The aim of this study was to assess the impact of cardiac hybrid imaging on the choice of treatment strategy selection for CAD.

Methods and Results Three hundred and eighteen consecutive patients underwent a 1-day stress/rest ${ }^{99 m}$ Tc-tetrofosmin SPECT and a CCTA on a separate scanner for evaluation of CAD. Patients were divided into one of the following three groups according to findings in the hybrid images obtained by fusing SPECT and CCTA: (i) matched finding of stenosis by CCTA and corresponding reversible SPECT defect; (ii) unmatched CCTA and SPECT finding; (iii) normal finding by both CCTA and SPECT. Follow-up was confined to the first 60 days after hybrid imaging as this allows best to assess treatment strategy decisions including the revascularization procedure triggered by its findings. Hybrid images revealed matched, unmatched, and normal findings in 51, 74, and 193 patients. The revascularization rate within 60 days was 41, 11, and $0 \%$ for matched, unmatched, and normal findings, respectively ( $P<.001$ for all inter-group comparisons). Thus, cardiac hybrid imaging with SPECT and CCTA provides an added clinical value for decision making with regard to treatment strategy for CA.

Significance This interesting study reports the impact of combined anatomical and perfusion information obtained by hybrid imaging on the decision to revascularize patients with CAD.

Structural Abnormalities of the Coronary Arterial Wall-In Addition to Luminal Narrowing-Affect Myocardial Blood Flow Reserve

Pisa, Italy and Genoa Italy

Ligra R, Marini C, Coceani M, Filidei E, Schueter M, Bianchi M, Rossi G, Pardini S, Salvadori P, Pardi O, Rovai D, Sambuceti G, Marraccini P, Neglia D J Nucl Med 2011; 52: 1704-1712

Context Multiple other factors in addition to obstructive CAD are known to affect myocardial blood flow (MBF) reserve. These investigators sought to explore the effects of coronary luminal narrowing, structural abnormalities of the coronary arterial wall, and cardiovascular risk factors on regional and global MBF reserve.

Methods and Results They studied 68 patients (mean age $\pm \mathrm{SD}, 61 \pm 10$ years; 41 men, 27 women) with an intermediate probability of coronary artery disease. The severity of coronary stenoses and the fibroadipose, fibromuscular, and calcium components of the coronary arterial wall were measured by 64-row multislice CT coronary angiography. Regional and global MBF reserve was measured by PET using ${ }^{13} \mathrm{~N}$-ammonia as a flow tracer at rest and after dipyridamole. One or more significant coronary stenoses ( $\geq 50 \%$ luminal narrowing) were present in 32 patients $(47 \%)$, and nonsignificant stenoses were present in 15 patients $(22 \%)$. Regional MBF reserve was significantly different in the territories perfused by normal coronary arteries, nonsignificant coronary stenoses, and significant coronary stenoses $(P<.001)$. Calcium content was higher in the coronary arteries with significant or nonsignificant stenoses $(0.95 \% \pm 1.08 \%$ and $0.73 \% \pm 0.93 \%$, respectively) than in those without stenoses $(0.11 \% \pm 0.38 \%, P<.001)$. Significant coronary stenosis $(P=.047)$ and calcium content $(P=.017)$ were the only independent determinants of impaired regional $\mathrm{MBF}$ reserve using multivariate analysis. At multiple logistic regression analysis, the Framingham risk score, an index of global cardiovascular risk burden, was the only significant determinant of global $\mathrm{MBF}$ reserve $(P=.028)$. Thus, coronary stenoses and coronary calcium content independently affect regional MBF reserve. Framingham risk score is the only significant determinant of global MBF reserve.

Significance This study shows that an abnormal coronary wall structure, in addition to the presence and the severity of coronary stenosis, affects regional MBF. 
Investigating Vulnerable Atheroma Using Combined ${ }^{18}$ F-FDG PET/CT Angiography of Carotid Plaque with Immunohistochemical Validation

London, United Kingdom and Brighton, United Kingdom

Menezes L, Kotze CW, Agu O, Richards T, Brookes J, Goh VJ, Rodriquez-Justo M, Endozo R, Harvey R, Yusuf SW, Ell PJ, Grooves AM

Journal of Nuclear Medicine 2011; 52: 1689-1703

Context To investigate the contribution of inflammation and angiogenesis to plaque vulnerability, and of calcification to plaque stability in vivo, these investigators combined CT angiography and PET and compared the findings with immunohistochemistry for patients undergoing carotid endarterectomy.

Methods and Results Twenty-one consecutive patients (18 men, 3 women; mean age $\pm \mathrm{SD}, 68.3 \pm 7.3$ ) undergoing carotid endarterectomy were recruited for combined carotid ${ }^{18}$ F-FDG PET/CT angiography. Plaque ${ }^{18}$ F-FDG uptake was quantified with maximum standardized uptake value, and CT angiography quantified percentage plaque composition (calcium and lipid). Surgical specimens underwent ex vivo CT aiding image registration, followed by immunohistochemical staining for CD68 (macrophage density) and vascular endothelial growth factor (angiogenesis). Relationships between imaging and immunohistochemistry were assessed with Spearman rank correlation and multivariable regression. The mean $( \pm \mathrm{SD})$ surgically excised carotid plaque ${ }^{18}$ F-FDG metabolism was $2.4( \pm 0.5)$ versus $2.2( \pm 0.3)$ contralaterally $(P=.027)$. There were positive correlations between plaque ${ }^{18} \mathrm{~F}$-FDG metabolism and immunohistochemistry with $\operatorname{CD68}(\rho=0.55 ; P=.011)$ and vascular endothelial growth factor $(\rho=0.47$; $P=.031)$. There was an inverse relationship between plaque ${ }^{18}$ F-FDG metabolism and plaque percentage calcium composition on $\mathrm{CT}(\rho=-0.51 ; P=.018)$ and between calcium composition and immunohistochemistry with CD68 $(\rho=-0.57 ; P=.007)$. Regression showed that maximum standardized uptake value and calcium composition were independently significant predictors of angiogenesis, and calcium composition was a predictor of macrophage density.

Significance This study provides in vivo evidence that increased plaque metabolism is associated with increased biomarkers of angiogenesis and inflammation, whereas plaque calcification is inversely related to PET and histologic biomarkers of inflammation.
Atherosclerosis Plaque Heterogeneity and Response to Therapy Detected by In Vivo Molecular Imaging of Matrix Metalloproteinase Activation

New Haven, CT and North Billerica, MA

Razavian M, Tavakoli S, Zhang J, Nie L, Dobrucki L, Sinusas AJ, Azure M, Robinsosn S, Sadeghi M

Journal of Nuclear Medicine 2011; 52: 1795-1802

Context Matrix metalloproteinases (MMPs) play a key role in the development of atherosclerosis and its complications. In vivo detection and quantification of MMP activation can help track the propensity to complications and response to therapy. These investigators sought to establish an in vivo imaging approach for monitoring MMP activation in atherosclerotic mouse aorta and use it to assess the response to dietary modification.

Method and Results Apolipoprotein-deficient mice were fed normal chow or a high-fat diet (HFD) for up to 3 months or a HFD for 2 months, followed by 1 month on normal chow. Then they underwent micro-SPECT/ $\mathrm{CT}$, along with autoradiography and oil red $\mathrm{O}$ staining of tissues. After 3 months of HFD, there was considerable atherosclerosis in the aorta. In vivo micro-SPECT/CT using RP782 (an ${ }^{111}$ in-labeled tracer targeting activated MMPs) showed a heterogeneous pattern of tracer uptake along the aorta. Heterogeneity of RP782 uptake was confirmed by autoradiography, and specificity was demonstrated using excess unlabeled precursor. Tracer uptake quantified by micro-SPECT significantly correlated with uptake quantified by autoradiography. Comparison of oil red $\mathrm{O}$ staining with autoradiography demonstrated areas of discordance between plaque presence and tracer uptake. HFD withdrawal led to significant reduction in RP782 uptake beyond the effect on plaque area. MMP expression and macrophage infiltration were similarly heterogeneous along the aorta and significantly reduced after withdrawal from the HFD. Finally, RP782 uptake significantly correlated with aortic macrophage content. Thus, molecular imaging of MMP activation reveals the heterogeneity of atherosclerotic plaques and is a useful tool for tracking plaque biology and response to therapy.

Significance This study demonstrates the feasibility and utility of micro-SPECT/CT for in vivo imaging of MMP activity in atherosclerotic plaques. 